\title{
Introduction to the special issue on adrenal imaging
}

\author{
Khaled M. Elsayes ${ }^{1}$
}

Published online: 9 March 2020

(c) Springer Science+Business Media, LLC, part of Springer Nature 2020
This special issue of Abdominal Radiology is dedicated to imaging of the adrenal gland. The articles included herein cover a broad range of topics, with the goal being to provide a comprehensive overview of the state-of-the-art knowledge related to adrenal imaging.

Adrenal masses are usually discovered incidentally on imaging performed for unrelated conditions. The prevalence of these incidentalomas have increased significantly with the increasing use of various cross-sectional imagings. The vast majority of adrenal incidentalomas are benign, nonfunctioning, and clinically insignificant lesions. However, a comprehensive evaluation is recommended to exclude malignant and functioning lesions that may require treatment, such as adrenocortical cancer, adrenal metastases, and pheochromocytoma, and to determine which masses require further testing and management.

In this special issue, a wide spectrum of adrenal pathologies in both the pediatric and adult populations are discussed in articles written by leading radiologists and experts from multiple specialties in the field. What emerges is a comprehensive systematic overview of the varying pathologies involving the adrenal glands and the crucial role imaging plays in the differentiation of malignant from benign lesions, which can be challenging for both non-radiologist clinicians and radiologists. We highlight the role of various imaging modalities in the evaluation of adrenal masses and their typical and atypical imaging features in concert with careful clinical and biochemical evaluation, which plays a crucial role in determining the subsequent management of these masses.
Also discussed in this issue are the standard imaging protocols and the current guidelines for the evaluation of adrenal masses, as well as the recent changes to the guidelines made by the American College of Radiology (ACR). Applying these guidelines requires close collaboration between physicians from multiple specialties, including radiology, endocrinology, and surgery, to provide patients with the optimum management options. By following current recommendations, radiologists can optimize patients care by efficiently determining which pathologies require further workup.

Additionally, we explore the potential pitfalls in the imaging of adrenal glands that result in misdiagnosis of adrenal masses, including the inappropriate choice of imaging technique, presence of pseudolesions, and overlap of imaging features of various adrenal pathologies.

I am honored to serve as the guest editor for this issue and would like to thank all the authors for sharing their expertise and contributing to this project. I would also like to thank the editor-in-chief, Dr. C. Daniel Johnson for inviting me to serve in this role. I am confident that the articles included in this issue will help practicing physicians and radiologists have a better understanding of the current guidelines and recommendations regarding the diagnosis and management of adrenal pathologies and, thus, provide their patients with the best possible care.

Publisher's Note Springer Nature remains neutral with regard to jurisdictional claims in published maps and institutional affiliations.
Khaled M. Elsayes

kmelsayes@mdanderson.org

1 Department of Diagnostic Radiology, The University of Texas MD Anderson Cancer Center, 1400 Pressler Street, Houston, TX 77030, USA 